\title{
Use of Technology to Support Information Needs for Continuity of Operations Planning in Public Health: A Systematic Review
}

\author{
Blaine Reeder ${ }^{1}$, Anne Turner ${ }^{1}$, George Demiris ${ }^{1}$ \\ ${ }^{1}$ Department of Medical Education and Biomedical Informatics, University of Washington, \\ Seattle, WA, USA 98195
}

Abstract

Objectives: Continuity of operations planning focuses on an organization's ability to deliver essential services before, during and after an emergency. Public health leaders must make decisions based on information from many sources and their information needs are often facilitated or hindered by technology. The aim of this study is to provide a systematic review of studies of technology projects that address public health continuity of operations planning information needs and to discuss patterns, themes, and challenges to inform the design of public health continuity of operations information systems.

Methods: To return a comprehensive results set in an under-explored area, we searched broadly in the Medline and EBSCOHost bibliographic databases using terms from prior work in public health emergency management and continuity of operations planning in other domains. In addition, we manually searched the citation lists of publications included for review.

Results: A total of 320 publications were reviewed. Twenty studies were identified for inclusion (twelve risk assessment decision support tools, six network and communications-enabled decision support tools, one training tool and one dedicated video-conferencing tool). Levels of implementation for information systems in the included studies range from proposed frameworks to operational systems.

Conclusion: There is a general lack of documented efforts in the scientific literature for technology projects about public health continuity of operations planning. Available information about operational information systems suggest inclusion of public health practitioners in the design process as a factor in system success.

Keywords: Public Health; Continuity of operations; Business continuity, Emergency management; Information systems; Technology 


\section{Introduction}

Disasters such as Hurricane Katrina and Hurricane Rita of 2005, the terrorist attacks on September 11, 2001 and the recent H1N1 influenza threat have raised awareness of the role of public health in emergency management in the United States in the last decade. An in-place and up-to-date continuity of operations plan (COOP) is an important part of any disaster preparedness and response strategy as recognized by emergency management experts ${ }^{[1-3]}$. A $\mathrm{COOP}$-- also known as a business continuity plan (BCP) -- provides guidelines for an organization to sustain delivery of essential services before, during and after a crisis. However, given that "state and local public health agencies lack sufficient staff and resources to manage their multiple preparedness responsibilities simultaneously" ${ }^{\text {[4] }}$, technology that supports COOP decision-making information needs during a crisis may be lacking within the overall emergency management strategies of many local public health departments. A further complication is that COOP efforts and emergency response efforts compete for the same organizational resources with the potential risk that emergency response may deplete resources necessary to maintain continuity of operations in public health.

Rozek and Groth, in their discussion of COOP for health care organizations, state that it "is no longer just a phase or project to be implemented when time and resources allow. It must be an ongoing program implemented to protect data, and ensure the integrity and security of the total organization". ${ }^{[1]}$ This need is also reflected in the work of Dawes et al. who interviewed 29 people directly involved in the World Trade Center (WTC) response and found that "[m]ost interviewees urged much greater attention to continuity of operations and disaster recovery"; however, in spite of that need they observe that "most small businesses, nonprofit organizations, and local governments remain have-nots in terms of resources, technology, preparedness, and response and recovery capability." ${ }^{[2]}$

Our experiences since January 2008, while documenting public health leadership decisionmaking information needs at a large municipal public health agency, confirm a lack of decision support technology for continuity of operations planning within the public health domain. ${ }^{[5]}$ In addition, Somers finds that "remarkably little attention has been paid to the documentation of local government COOP planning efforts" and cites a FEMA [Federal Emergency Management Agency] report that states the "ability of an agency to execute its essential functions ... is dependent upon the identification, availability, and redundancy of critical communications and information technology systems to support key government leadership, internal elements, other agencies, critical customers, and the public". ${ }^{[3]}$ Fraser remarks on recent public health budget cuts and observes a lack of standard preparedness measures and assessment criteria. He concludes that the United States may not be ready for a pandemic or disaster. ${ }^{[6]}$ Taken together, these findings indicate a need for information technology to support public health COOP activities but also show that this critical need may be unaddressed or not fully recognized in practice.

Technology must address the information needs of public health COOP planners and practitioners to successfully support their work processes and decision-making activities. In order to assess technology support of public health COOP, we compiled a list of information needs ${ }^{[7]}$ suggested by prior work in public health knowledge management ${ }^{[8]}$, COOP planning ${ }^{[6,9-}$ 
${ }^{11]}$, post-disaster studies of health care worker information needs ${ }^{[12,13]}$, public health preparedness ${ }^{[14-18]}$ and disaster planning ${ }^{[19-21]}$. The information needs on this list are included based on author familiarity with a broad corpus of public health and continuity of operations literature gained through searches of the literature over time and experiences of the authors working with public health continuity of operations planners since January 2008. This list represents broad categories of information needs for public health COOP and may not represent every information need available from the published literature. In addition to these information needs, there is a basic requirement that decision-making information be available and up-to-date at all times before, during and after a crisis. These information needs are:

- $\quad$ synthesized information ${ }^{[8]}$

- centralized data access ${ }^{[8]}$

- coordination/incident command support ${ }^{[6,9-21]}$

- $\quad$ staff training/education ${ }^{[6,9-21]}$, planning

- plan testing/exercise support $^{[6,9-17,20,21]}$

- interoperable external communication/alerting ${ }^{[6,9,11-21]}$

- internal communication/alerting ${ }^{[6,9,11-17,19,21]}$

- staff attendance/contact list management $^{[6,9,11,13-17,19,21]}$

- resource tracking/capacity management ${ }^{[6,11,13-17,19-21]}$

- collaboration $^{[8,11-14,16,17,19-21]}$

- remote work/portable data ${ }^{[6,11,17-19,21]}$

- geographic data ${ }^{[11,13]}$

\section{Objectives}

Previous technology efforts in the area of public health can inform the identification of system features that support public health continuity of operations planning and emergency decision support. Furthermore, it is important to explore technologies, approaches and challenges to advance the design of future public health COOP information systems. The aim of this paper is to identify and review published studies of information systems and technology projects that address information needs in the context of public health continuity operations planning and review public health emergency management information systems that are applicable to public health COOP.

\section{Methods}

We conducted a systematic literature review of studies of public health continuity of operations planning technology projects. However, as public health COOP has not been studied extensively, we searched broadly using terms from our categories of information needs compiled from publications about public emergency management and private continuity of operations planning. These terms were used in combination with the terms "public health", "disaster", "emergency", "technology", "information system", "informatics" and "decision support". We conducted our initial search in the Medline bibliographic database as well as in EBSCOhost from January 1990 to January 2009, using the same term combinations, in January of 2009. Based on this strategy, results from a search of both bibliographic databases yielded a total of 469 articles (Medline: 
213, EBSCOhost: 256). In addition, we reviewed the table of contents for all issues of the Journal of Business Continuity and Emergency Planning, because of its specialized focus that is of high relevance to our study. All citations were imported into a commercial reference manager.

Three reviewers rated ten percent of the article abstracts (thirty-two) to validate inclusion/exclusion criteria. After a high degree of agreement between reviewers was determined at a face-to-face meeting, the remaining articles were rated by the first author. Reference lists for each included article were reviewed for other studies that might be eligible for inclusion. In addition, information systems discovered during the full-text review of each article were noted and searches for studies of these systems were conducted. The process followed during the literature review is illustrated in Figure 1.

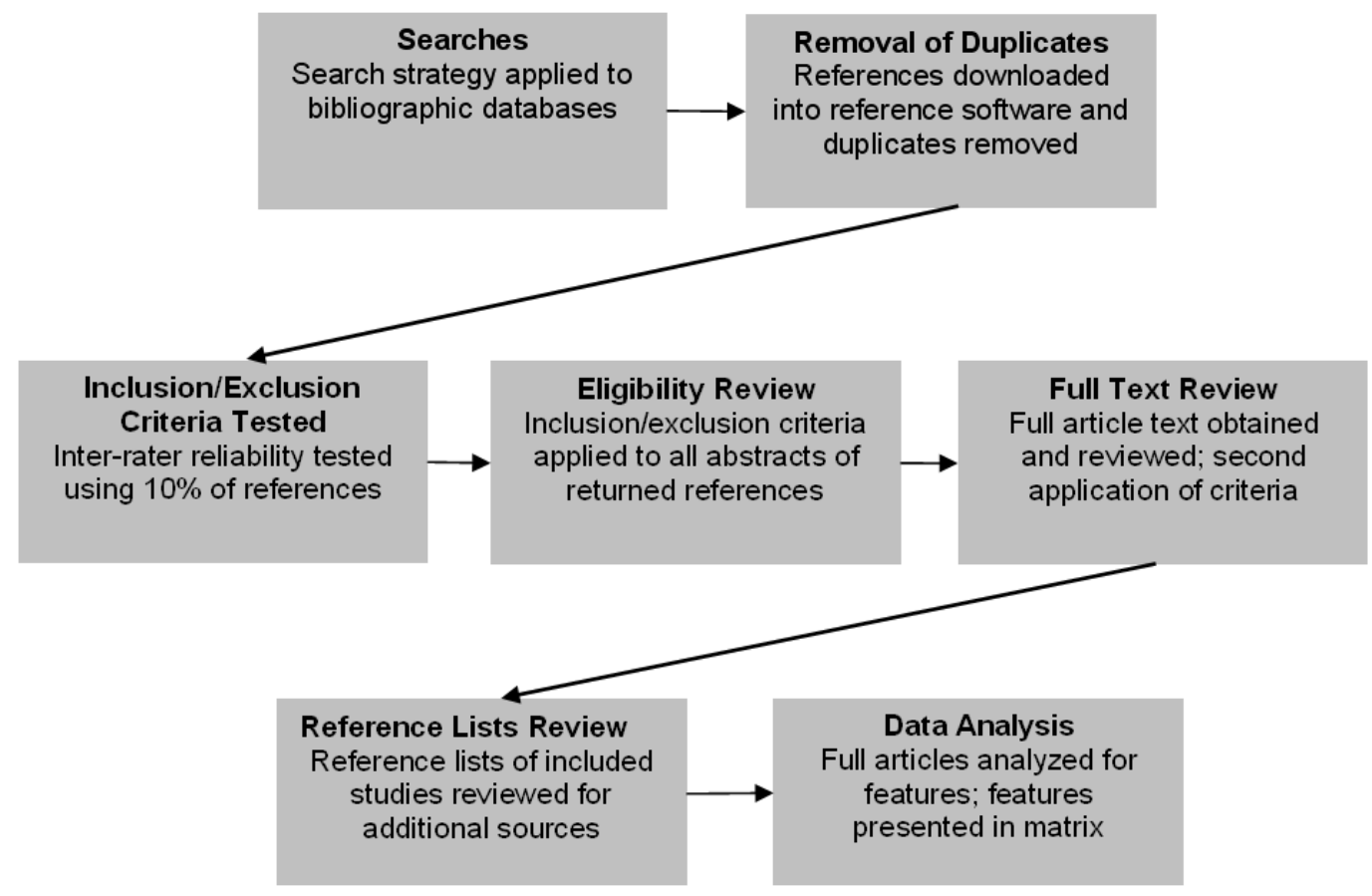

\section{Figure 1. Process flow of literature survey methods from search strategy to data analysis}

Inclusion criteria included studies of technologies that were developed specifically to support continuity of operations planning information needs. In addition, inclusion criteria included studies of technologies developed for general emergency management purposes that implement features or approaches that could support continuity of operations planning information needs. Our inclusion criteria were defined to encompass both specific continuity of operations planning systems and general emergency management systems to broaden the number of potential systems and technologies included for analysis.

Exclusion criteria included articles that focus mainly on public health data stores, registries and general technology infrastructure as well as those that discuss general public health policy, emergency management policy or continuity of operations planning but do not reference or evaluate a specific system or systems. In addition, exclusion criteria included articles that discuss studies, reports and evaluations of public health interventions, live training exercises, general 
computer training and efforts to improve quality of service lines. Lastly, exclusion criteria included studies of safety monitoring, disease surveillance, clinical or laboratory information systems and work flow in specific settings not related to public health. As our search strategy is necessarily comprised of terms that make it somewhat broad in order to return a result set that includes the systems and technologies we are targeting, our list of exclusion criteria was very detailed and specific.

\section{Results}

After duplicates were removed in the commercial reference manager, a total of 320 articles remained and were further reviewed to determine inclusion eligibility. Twenty studies of information systems and technology projects met our public health continuity of operations planning information needs inclusion/exclusion criteria. Included studies were published between 1995 to 2008. Most included studies originated from the United states (13) but some included studies originated from Greece (2), Canada (1), Mexico (1), Spain (1), Turkey (1) and the United Kingdom (1). This list of systems and projects is by no means an exhaustive list of such systems worldwide but is a list of systems and projects that have reported details of design, implementation or findings published in scientific indexed literature and met our criteria for inclusion. Table I presents the details and a short description of each system or project.

\section{Discussion}

The concept of technology specifically designed to serve public health continuity of operations planning information needs is relatively novel and, indeed, only one system in the included studies (Access Grid $^{[22]}$ ) explicitly mentions continuity of operations (as "business continuity") in the context of public health. The inclusion of only one such study is not surprising in that, as we have previously observed, continuity of operations planning and information technology to support it, are underexplored in the public health domain. This apparent lack of studies of public health continuity of operations information systems and technology projects presents many opportunities for informatics research, including the design of integrated systems that support routine public health processes and remote access to data. However, awareness of continuity of operations as a component that competes for public emergency management resources must be raised and addressed at an organizational level before such informatics efforts can be successful. 
Use of Technology to Support Information Needs for Continuity of Operations Planning in Public Health: A Systematic Review

Table 1. Selected System Features and Details

\begin{tabular}{|c|c|c|c|c|c|}
\hline $\begin{array}{c}\text { System } \\
\text { Name \& } \\
\text { Study Authors }\end{array}$ & $\begin{array}{l}\text { Pub. } \\
\text { Year }\end{array}$ & System Features and Details & $\begin{array}{l}\text { Level of } \\
\text { Implementation }\end{array}$ & $\begin{array}{l}\text { Method or } \\
\text { Technique }\end{array}$ & $\begin{array}{l}\text { Funding } \\
\text { Source }\end{array}$ \\
\hline $\begin{array}{l}\text { Emergency } \\
\text { Computer } \\
\text { Communicat } \\
\text { ions } \\
\text { Network } \\
\text { (CDC } \\
\text { Wonder/PC) } \\
\text { O'Carroll, et } \\
\text { al. }{ }^{[23]}\end{array}$ & 1995 & $\begin{array}{l}\text { - Possibly the first } \\
\text { electronic public health } \\
\text { system of its kind in a } \\
\text { disaster relief operation } \\
\text { - Local data entry with } \\
\text { secure e-mail transmission } \\
\text { of database files } \\
\text { - Demonstrated the } \\
\text { effectiveness of digital } \\
\text { communication in reducing } \\
\text { the impact of a disaster }\end{array}$ & $\begin{array}{l}\text { Operational } \\
\text { System }\end{array}$ & $\begin{array}{l}\text { CDC conducted } \\
\text { consultations with } \\
\text { Iowa health } \\
\text { department } \\
\text { officials about } \\
\text { implementation. } \\
11 \text { two person } \\
\text { teams installed } \\
\text { systems and } \\
\text { trained staff for } \\
99 \text { county } \\
\text { installations. }\end{array}$ & $\begin{array}{l}\text { Stafford } \\
\text { Act funds } \\
\text { authorized } \\
\text { by the } \\
\text { Federal } \\
\text { Emergency } \\
\text { Manageme } \\
\text { nt Agency }\end{array}$ \\
\hline $\begin{array}{l}\text { EPEDAT } \\
\text { Eguchi, et } \\
\text { al. }^{[24]}\end{array}$ & 1997 & $\begin{array}{l}\text { - Integrates real-time and } \\
\text { historical earthquake data } \\
\text { - Provides loss- } \\
\text { estimation methods for } \\
\text { earthquake damage } \\
\text { - Applicable to a variety } \\
\text { of regions and users }\end{array}$ & $\begin{array}{l}\text { Partially } \\
\text { functional } \\
\text { system }\end{array}$ & $\begin{array}{l}\text { Iteratively links } \\
\text { earthquake data } \\
\text { source parameters } \\
\text { and damage } \\
\text { algorithms. } \\
\text { Displays output } \\
\text { using GIS } \\
\text { technology. }\end{array}$ & $\begin{array}{l}\text { California } \\
\text { Governor's } \\
\text { Office of } \\
\text { Emergency } \\
\text { Services }\end{array}$ \\
\hline $\begin{array}{l}\text { ICEM-SE } \\
\text { Project } \\
\text { Bedard, et } \\
\text { al. }^{[25]}\end{array}$ & 2003 & $\begin{array}{l}\text { - Integrates GIS with } \\
\text { OLAP (On-line Analytical } \\
\text { Processing) for decision } \\
\text { support } \\
\text { - Developed for } \\
\text { environmental health } \\
\text { interventions and outcomes } \\
\text { - Could be applied to any } \\
\text { planning data source for } \\
\text { COOP planning }\end{array}$ & Prototype & $\begin{array}{l}\text { Integrates } \\
\text { commercially } \\
\text { available software } \\
\text { to simplify } \\
\text { geographic } \\
\text { visualization of } \\
\text { environmental } \\
\text { health data and } \\
\text { then analyzing } \\
\text { prototypes by } \\
\text { comparing task } \\
\text { steps }\end{array}$ & $\begin{array}{l}\text { Several } \\
\text { Canadian } \\
\text { Governme } \\
\text { nt funding } \\
\text { agencies }\end{array}$ \\
\hline $\begin{array}{l}\text { GEPIMI } \\
\text { Ptochos, et } \\
\text { al. }{ }^{[26]}\end{array}$ & 2004 & $\begin{array}{l}\text { Developed to establish } \\
\text { a public health network in } \\
\text { Greece, Bulgaria, Albania, } \\
\text { Fyrom and Turkey } \\
\text { - Integrates medical, }\end{array}$ & Pilot System & $\begin{array}{l}\text { Integrates } \\
\text { multiple data } \\
\text { sources using } \\
\text { many different } \\
\text { technologies and }\end{array}$ & $\begin{array}{l}\text { Greek } \\
\text { Ministry of } \\
\text { Health and } \\
\text { Welfare } \\
\text { and the }\end{array}$ \\
\hline
\end{tabular}




\begin{tabular}{|c|c|c|c|c|c|}
\hline & & $\begin{array}{l}\text { epidemiological and } \\
\text { environmental data with } \\
\text { GIS technology } \\
\text { - Accesses and } \\
\text { standardizes information } \\
\text { from numerous systems }\end{array}$ & & $\begin{array}{l}\text { provides data } \\
\text { display via the } \\
\text { Web and GIS. }\end{array}$ & $\begin{array}{l}\text { European } \\
\text { Commissio } \\
\mathrm{n}\end{array}$ \\
\hline $\begin{array}{l}\text { Maxi-Vac } \\
\text { Washington, } \\
\text { et al. }\end{array}$ & 2005 & $\begin{array}{l}\text { - Developed for mass } \\
\text { vaccination planning } \\
\text { support through } \\
\text { simulations } \\
\text { - Can be used in a variety } \\
\text { of settings } \\
\text { - Facilitates staff } \\
\text { management based on } \\
\text { service demand }\end{array}$ & $\begin{array}{l}\text { Operational } \\
\text { system for a } \\
\text { small-pox } \\
\text { vaccination } \\
\text { scenario in a } \\
\text { hypothetical } \\
\text { clinic } \\
\text { developed } \\
\text { with user } \\
\text { input }\end{array}$ & $\begin{array}{l}\text { Integrates several } \\
\text { commercially } \\
\text { available } \\
\text { modeling tools, } \\
\text { data sources and } \\
\text { expert opinion to } \\
\text { determine staff } \\
\text { utilization }\end{array}$ & $\begin{array}{l}\text { Centers for } \\
\text { Disease } \\
\text { Control }\end{array}$ \\
\hline $\begin{array}{l}\text { Arcview } 3.2 \\
\text { with } 2000 \\
\text { Census data } \\
\text { Waring, et } \\
\text { al. }{ }^{[28]}\end{array}$ & 2005 & $\begin{array}{l}\text { - GIS technology applied } \\
\text { to rapid needs assessment } \\
\text { for disasters in the } \\
\text { Houston, TX } \\
\text { - A method of using } \\
\text { existing GIS software tools } \\
\text { and data sources } \\
\text { - First study of its kind in } \\
\text { the Houston area }\end{array}$ & $\begin{array}{l}\text { Pilot system } \\
\text { tested during } \\
\text { the aftermath } \\
\text { of a tropical } \\
\text { storm }\end{array}$ & $\begin{array}{l}\text { Employs an } \\
\text { integrated GIS } \\
\text { and data } \\
\text { methodology that } \\
\text { combines } \\
\text { commercial } \\
\text { software with } \\
\text { available year } \\
2000 \text { census data }\end{array}$ & $\begin{array}{l}\text { University } \\
\text { of Texas } \\
\text { Center for } \\
\text { Public } \\
\text { Health and } \\
\text { Policy } \\
\text { Studies } \\
\text { (CHAMPS } \\
\text { ) }\end{array}$ \\
\hline $\begin{array}{l}\text { DSS-DM } \\
\text { Aleskerov, et } \\
\text { al. }^{[30]}\end{array}$ & 2005 & $\begin{array}{l}\text { - Flexible simulation of } \\
\text { various earthquake disaster } \\
\text { scenarios based on } \\
\text { different inputs } \\
\text { - Developed for planning } \\
\text { and mitigation purposes } \\
\text { - Provides micro-level } \\
\text { estimates of human losses } \\
\text { and damage for better } \\
\text { decision support }\end{array}$ & $\begin{array}{l}\text { Proposed } \\
\text { system } \\
\text { developed and } \\
\text { tested with } \\
\text { user } \\
\text { involvement }\end{array}$ & $\begin{array}{l}\text { Uses PC-based } \\
\text { commercial } \\
\text { software (MS } \\
\text { Access and } \\
\text { Delphi) and data } \\
\text { from district and } \\
\text { sub-district } \\
\text { administrators to } \\
\text { produce micro- } \\
\text { level loss } \\
\text { estimations for } \\
\text { building clusters }\end{array}$ & $\begin{array}{l}\text { Bogaziçi } \\
\text { University, } \\
\text { Istanbul, } \\
\text { Turkey }\end{array}$ \\
\hline
\end{tabular}


Use of Technology to Support Information Needs for Continuity of Operations Planning in Public Health:

A Systematic Review

Table 1. Selected System Features and Details

\begin{tabular}{|c|c|c|c|c|c|}
\hline $\begin{array}{c}\text { System Name } \\
\text { \& Study } \\
\text { Authors }\end{array}$ & $\begin{array}{l}\text { Pub. } \\
\text { Year }\end{array}$ & System Features and Details & $\begin{array}{l}\text { Level of } \\
\text { Implementation }\end{array}$ & $\begin{array}{l}\text { Method or } \\
\text { Technique }\end{array}$ & $\begin{array}{l}\text { Funding } \\
\text { Source }\end{array}$ \\
\hline $\begin{array}{l}\text { RealOpt } \\
\text { Lee, et al. }{ }^{[31]}\end{array}$ & 2006 & $\begin{array}{l}\text { - Rapid simulation } \\
\text { system for infectious } \\
\text { disease disaster scenario } \\
\text { effects on populations for } \\
\text { real-time decision support } \\
\text { about resource allocation } \\
\text { - Allows for flexible } \\
\text { "what-if" configuration } \\
\text { - Allows users to create } \\
\text { floor plans and specify } \\
\text { inputs }\end{array}$ & $\begin{array}{l}\text { Operational } \\
\text { system } \\
\text { implemented } \\
\text { with staffing } \\
\text { information } \\
\text { from an } \\
\text { Anthrax field } \\
\text { exercise. }\end{array}$ & $\begin{array}{l}\text { Tests simulations } \\
\text { of staffing for } \\
\text { medication } \\
\text { dispensing in } \\
\text { small pox } \\
\text { scenarios and } \\
\text { compares the } \\
\text { results against a } \\
\text { commercial } \\
\text { system that uses } \\
\text { the same model }\end{array}$ & $\begin{array}{l}\text { Institute of } \\
\text { Technolog } \\
\text { y, Atlanta, } \\
\text { GA/ } \\
\text { Centers for } \\
\text { Disease } \\
\text { Control }\end{array}$ \\
\hline $\begin{array}{l}\text { ACCESS } \\
\text { Grid } \\
\text { Hauenstein, } \\
\text { et al. }{ }^{[22]}\end{array}$ & 2006 & $\begin{array}{l}\text { - Demonstrates Access } \\
\text { Grid videoconferencing as } \\
\text { an approach to } \\
\text { transnational } \\
\text { communications } \\
\text { - Directly addresses } \\
\text { public health business } \\
\text { continuity collaboration } \\
\text { information need } \\
\text { - Requires additional } \\
\text { participant installations and } \\
\text { testing to be a full solution }\end{array}$ & $\begin{array}{l}\text { Operational } \\
\text { system }\end{array}$ & $\begin{array}{l}\text { Evaluated during } \\
\text { a } 210 \text { minute test } \\
\text { of a video- } \\
\text { conference } \\
\text { platform through } \\
\text { participation of } \\
\text { ten Asia Pacific } \\
\text { Economic } \\
\text { Cooperation } \\
\text { Emerging } \\
\text { Infections } \\
\text { Network (APEC } \\
\text { EINet) on January } \\
\text { 19, 2006. }\end{array}$ & $\begin{array}{l}\text { APEC, } \\
\text { IBM and } \\
\text { the Global } \\
\text { Health and } \\
\text { Securities } \\
\text { Initiative, } \\
\text { NTI }\end{array}$ \\
\hline $\begin{array}{l}\text { ToxMap } \\
\text { Hauenstein, } \\
\text { et al. }{ }^{[32]}\end{array}$ & 2006 & $\begin{array}{l}\text { NLM system that } \\
\text { integrates EPA hazardous } \\
\text { chemical data with GIS } \\
\text { data } \\
\text { - Creates dynamic maps } \\
\text { of chemical releases, } \\
\text { trends, facility locations } \\
\text { and superfund sites } \\
\text { - Provides links to } \\
\text { relevant information }\end{array}$ & $\begin{array}{l}\text { Operational } \\
\text { system } \\
\text { avalaible } \\
\text { online at } \\
\text { http://toxmap. } \\
\text { nlm.nih.gov/t } \\
\text { oxmap/main/i } \\
\text { ndex.jsp }\end{array}$ & $\begin{array}{l}\text { Integrates Toxic } \\
\text { Release Inventory } \\
\text { (TRI) data and } \\
\text { Web-based GIS } \\
\text { technology }\end{array}$ & $\begin{array}{l}\text { National } \\
\text { Library of } \\
\text { Medicine }\end{array}$ \\
\hline
\end{tabular}


Use of Technology to Support Information Needs for Continuity of Operations Planning in Public Health: A Systematic Review

\begin{tabular}{|c|c|c|c|c|c|}
\hline $\begin{array}{l}\text { AID-N } \\
\text { Hauenstein, } \\
\text { et al. }^{[33]}\end{array}$ & 2006 & $\begin{array}{l}\text { - Allows data access } \\
\text { through Web services } \\
\text { - Implements data } \\
\text { standards for disaster } \\
\text { information exchange } \\
\text { - Designed with real- } \\
\text { time communication in } \\
\text { mind }\end{array}$ & $\begin{array}{l}\text { Test bed } \\
\text { shared data } \\
\text { model }\end{array}$ & $\begin{array}{l}\text { Creates federated } \\
\text { data models } \\
\text { housed in a } \\
\text { central server. } \\
\text { Exposes data } \\
\text { through web } \\
\text { services } \\
\text { architecture. }\end{array}$ & $\begin{array}{l}\text { National } \\
\text { Library of } \\
\text { Medicine }\end{array}$ \\
\hline $\begin{array}{l}\text { HERDS } \\
\text { Gotham, et } \\
\text { al. }^{[34]}\end{array}$ & 2007 & $\begin{array}{l}\text { - State-wide All-Hazards } \\
\text { Preparedness System in } \\
\text { New York State } \\
\text { - Archetypal Public } \\
\text { Health Preparedness } \\
\text { System } \\
\text { - Provides flexible } \\
\text { functionality during } \\
\text { planned drills and real } \\
\text { events }\end{array}$ & $\begin{array}{l}\text { Operational } \\
\text { system used } \\
\text { in actual } \\
\text { health events } \\
\text { including a } \\
\text { blackout and } \\
\text { a hurricane } \\
\text { readiness } \\
\text { activation. }\end{array}$ & $\begin{array}{l}\text { Uses a } \\
\text { collaborative } \\
\text { development } \\
\text { across local } \\
\text { health } \\
\text { jurisdictions. } \\
\text { Employs an all- } \\
\text { hazards approach. } \\
\text { Establishes a } \\
\text { single data } \\
\text { reporting stream } \\
\text { built upon } \\
\text { existing } \\
\text { infrastructure. }\end{array}$ & $\begin{array}{l}\text { New York } \\
\text { State } \\
\text { Departmen } \\
\text { t of Health }\end{array}$ \\
\hline $\begin{array}{l}\text { SAFE } \\
\text { Chronaki, et } \\
\text { al. }{ }^{[35]}\end{array}$ & 2007 & $\begin{array}{l}\text { - Integrates multiple } \\
\text { modes of communication } \\
\text { including satellites with a } \\
\text { mobile coordination center } \\
\text { - Uses satellite video as } \\
\text { one means of collaboration } \\
\text { - Models work flow of } \\
\text { user groups during a } \\
\text { typhoid outbreak }\end{array}$ & $\begin{array}{l}\text { Proposed } \\
\text { architecture }\end{array}$ & $\begin{array}{l}\text { Integrates satellite } \\
\text { communications } \\
\text { with existing } \\
\text { health } \\
\text { information data } \\
\text { sources, } \\
\text { videoconferencin } \\
\text { g and GIS } \\
\text { technology }\end{array}$ & $\begin{array}{l}\text { SAFE } \\
\text { consortium } \\
\text { and the } \\
\text { European } \\
\text { Space } \\
\text { Agency } \\
\text { (ESA) }\end{array}$ \\
\hline
\end{tabular}


Use of Technology to Support Information Needs for Continuity of Operations Planning in Public Health:

A Systematic Review

Table 1. Selected System Features and Details

\begin{tabular}{|c|c|c|c|c|c|}
\hline $\begin{array}{c}\text { System Name } \\
\text { \& Study } \\
\text { Authors } \\
\end{array}$ & $\begin{array}{l}\text { Pub. } \\
\text { Year }\end{array}$ & System Features and Details & $\begin{array}{l}\text { Level of } \\
\text { Implementation }\end{array}$ & $\begin{array}{l}\text { Method or } \\
\text { Technique }\end{array}$ & $\begin{array}{l}\text { Funding } \\
\text { Source }\end{array}$ \\
\hline $\begin{array}{l}\text { EXPLORIS } \\
\text { Marti, et } \\
\text { al. }^{[36]}\end{array}$ & 2008 & $\begin{array}{l}\text { - Prototype developed to } \\
\text { to estimate earthquake } \\
\text { damage on Tenerife } \\
\text { (Canary Islands) } \\
\text { - Uses a deterministic } \\
\text { simulation model of a } \\
\text { volcanic eruption } \\
\text { - Creates a vulnerability } \\
\text { map for casualties and } \\
\text { fatalities using spatial } \\
\text { analysis and GIS }\end{array}$ & $\begin{array}{l}\text { Prototype } \\
\text { tested for a } \\
\text { number of } \\
\text { specific sites } \\
\text { in Europe (all } \\
\text { sites not } \\
\text { disclosed in } \\
\text { study) }\end{array}$ & $\begin{array}{l}\text { Integrates } \\
\text { volcanic hazard, } \\
\text { building } \\
\text { typology and } \\
\text { population data } \\
\text { to estimate } \\
\text { casualties and } \\
\text { fatalities during } \\
\text { eruptions. }\end{array}$ & $\begin{array}{l}\text { European } \\
\text { Union }\end{array}$ \\
\hline $\begin{array}{l}\text { DDSS } \\
\text { Lozano- } \\
\text { Fuentes, et } \\
\text { al. }^{[37]}\end{array}$ & 2008 & $\begin{array}{l}\text { - Inexpensive GIS } \\
\text { solution to increase public } \\
\text { health capacity } \\
\text { - Developed for } \\
\text { decision-support in } \\
\text { combating vector-borne } \\
\text { disease } \\
\text { - Incorporates high } \\
\text { resolution satellite imagery } \\
\text { with disease data to display } \\
\text { city infrastructure } \\
\text { information }\end{array}$ & $\begin{array}{l}\text { Pilot } \\
\text { demonstration }\end{array}$ & $\begin{array}{l}\text { Uses satellite } \\
\text { imagery and } \\
\text { Google-Earth } \\
\text { feature-making } \\
\text { tools to develop } \\
\text { city } \\
\text { infrastructure } \\
\text { layers and } \\
\text { visualize vector- } \\
\text { borne disease } \\
\text { data }\end{array}$ & $\begin{array}{l}\text { Innovative } \\
\text { Vector } \\
\text { Control } \\
\text { Consortium }\end{array}$ \\
\hline $\begin{array}{l}\text { BRFSS + } \\
\text { GIS } \\
\text { Technology } \\
\text { Holt, et } \\
\text { al.[ }\end{array}$ & 2008 & $\begin{array}{l}\text { - Developed with needs } \\
\text { assessment of vulnerable } \\
\text { populations in mind } \\
\text { - Integrates population } \\
\text { and GIS data available } \\
\text { from the CDC } \\
\text { - Flexible for use in } \\
\text { different disaster scenarios } \\
\text { like hurricanes and } \\
\text { influenza outbreaks }\end{array}$ & $\begin{array}{l}\text { Sample } \\
\text { Assessment }\end{array}$ & $\begin{array}{l}\text { Integrates } \\
\text { BRFSS data and } \\
\text { GIS technology } \\
\text { to visually } \\
\text { display a derived } \\
\text { risk assessment } \\
\text { for a hurricane } \\
\text { landfall along } \\
\text { the East and Gulf } \\
\text { coasts }\end{array}$ & $\begin{array}{l}\text { Centers for } \\
\text { Disease } \\
\text { Control }\end{array}$ \\
\hline
\end{tabular}


Use of Technology to Support Information Needs for Continuity of Operations Planning in Public Health: A Systematic Review

\begin{tabular}{|c|c|c|c|c|c|}
\hline $\begin{array}{l}\text { HAZUS-MH } \\
\text { Ding, et } \\
\text { al. }{ }^{[39]}\end{array}$ & 2008 & $\begin{array}{l}\text { - Models flooding, } \\
\text { hurricanes and earthquakes } \\
\text { - Provides for standard } \\
\text { and customized estimations } \\
\text { - Developed to answer } \\
\text { needs of government } \\
\text { officials to estimate } \\
\text { disaster damage }\end{array}$ & $\begin{array}{l}\text { Operational } \\
\text { system that } \\
\text { has been in } \\
\text { use since the } \\
\text { late 1990s }\end{array}$ & $\begin{array}{l}\text { Validates the } \\
\text { flood model in } \\
\text { HAZUS-MH } \\
\text { during the Harris } \\
\text { County Risk } \\
\text { Assessment } \\
\text { Program } \\
\text { (HCRAP) in } \\
\text { Harris County, } \\
\text { Texas by } \\
\text { comparing } \\
\text { outputs against } \\
\text { local analyses }\end{array}$ & $\begin{array}{l}\text { Federal } \\
\text { Emergency } \\
\text { Managemen } \\
\text { t Agency }\end{array}$ \\
\hline $\begin{array}{l}\text { EPIMS } \\
\text { Senior and } \\
\text { Copley }^{[40]}\end{array}$ & 2008 & $\begin{array}{l}\text { - Developed to manage, } \\
\text { assess and monitor } \\
\text { information during } \\
\text { emergencies } \\
\text { - Allows for internal and } \\
\text { external communication } \\
\text { - Visualizes incident } \\
\text { response and allows access } \\
\text { to planning documents }\end{array}$ & $\begin{array}{l}\text { Operational } \\
\text { system used } \\
\text { during actual } \\
\text { emergencies }\end{array}$ & $\begin{array}{l}\text { Integrates } \\
\text { disparate data } \\
\text { sources to allow } \\
\text { data and } \\
\text { communication } \\
\text { access to all } \\
\text { users through } \\
\text { Web, GIS and } \\
\text { database } \\
\text { technologies }\end{array}$ & $\begin{array}{l}\text { Rotherham } \\
\text { Metropolita } \\
\text { n Borough } \\
\text { Council }\end{array}$ \\
\hline $\begin{array}{l}\text { BCMS } \\
\text { Sheth, et } \\
\text { al. }^{[41]}\end{array}$ & 2008 & $\begin{array}{l}\text { - Strictly focuses on } \\
\text { continuity of operations } \\
\text { planning } \\
\text { - Provides an integrated } \\
\text { view of data from multiple } \\
\text { sources } \\
\text { - Industry independent } \\
\text { and flexible for use during } \\
\text { different scenarios }\end{array}$ & $\begin{array}{l}\text { Proposed } \\
\text { system based } \\
\text { on user needs } \\
\text { and } \\
\text { involvement }\end{array}$ & $\begin{array}{l}\text { Technology } \\
\text { independent } \\
\text { model based on } \\
\text { software } \\
\text { maturity/capabili } \\
\text { ty models }\end{array}$ & $\begin{array}{l}\text { State of } \\
\text { Maryland } \\
\text { Judiciary }\end{array}$ \\
\hline
\end{tabular}




\section{Patterns in information need support}

Looking at Table II, we can make some observations about the information needs supported by a given system or technology project. All systems satisfied the need for synthesized information. This is not surprising in that information systems are often designed to integrate data while decision-making is often dependent upon data that provides better situational awareness through synthesis. In addition, all systems that satisfied the need for coordination/incident command support also satisfied the need for collaboration. Again, this is not surprising in that coordination and collaboration activities both require communication and information flow but coordination often applies a more directed hierarchical approach. All systems that satisfied the need for centralized data access for a variety of practitioner roles also satisfied the need for remote work capability and/or portable data. This situation is likely due to the relatively recent advent of networked data stores that are accessed through web technologies; both centralized data access and data access are inherent features of these widely adopted technologies.

Of the twenty included studies, only two (the Emergency Computer Communications Network $^{[23]}$ and EPEDAT ${ }^{[24]}$ ), were published before September 11, 2001 (1995 and 1997, respectively). Included studies after that begin in 2003. These results coincide with the substantial federal funding available in the United States for the research and design of technology to support emergency management and combat terrorism after the 9/11 terrorist attacks. The two year lag in publications from 2001 to 2003 likely indicates the start up time to design, develop and conduct research on a given information system or technology project. With the exception of two systems (NCCPHP Training Web site ${ }^{[29]}$ and AID-N framework ${ }^{[33]}$ ), nearly all systems satisfied the need for planning/plan testing/exercise support. This observation can also be explained by the push for preparedness in a post-9/11 world. In addition, with the exception of one system (NCCPHP Training Web site ${ }^{[2]}$ ), nearly all systems satisfied the need for resource tracking/capacity management. This can be attributed to the fact that resource and capacity information are necessary to support decision-making in an emergency. 
Use of Technology to Support Information Needs for Continuity of Operations Planning in Public Health:

A Systematic Review

Table 2. Public health continuity of operations information needs supported by included systems

\begin{tabular}{|c|c|c|c|c|c|c|c|c|c|c|c|c|c|c|}
\hline Author & $\begin{array}{l}\text { System Name } \\
\text { (Location) }\end{array}$ & $\begin{array}{l}\text { Year } \\
\text { of } \\
\text { Study } \\
\text { Pub. }\end{array}$ & 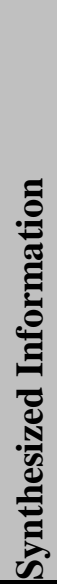 & 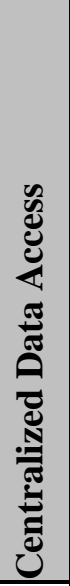 & 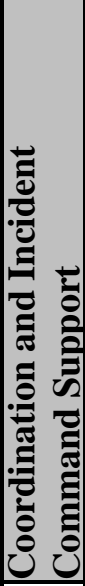 & 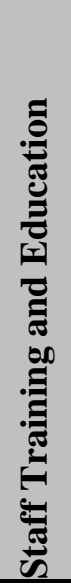 & 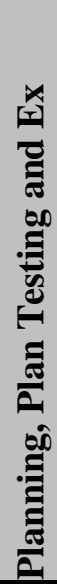 & 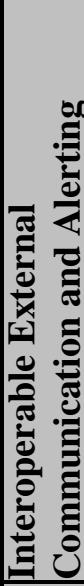 & 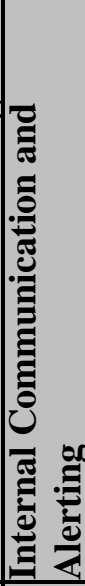 & 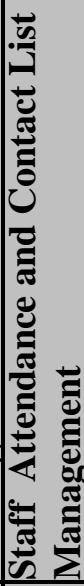 & 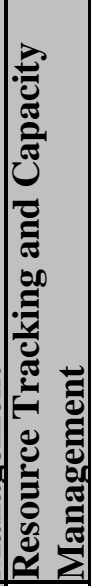 & 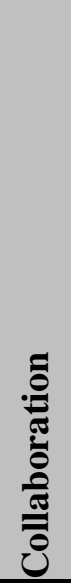 & 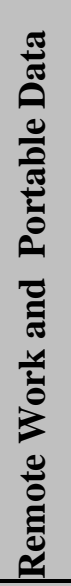 & 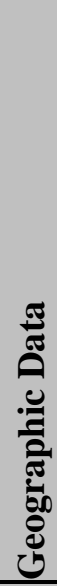 \\
\hline $\begin{array}{l}\text { Sheth, et } \\
\text { al. }{ }^{[41]}\end{array}$ & BCMS (UK) & 2008 & $\mathrm{X}$ & $\mathrm{X}$ & $\mathrm{X}$ & $\mathrm{X}$ & $X$ & & & & $\mathrm{X}$ & $\mathrm{X}$ & & \\
\hline $\begin{array}{l}\text { Holt, et } \\
\text { al. }{ }^{[38]}\end{array}$ & BRFSS (US) & 2008 & $X$ & & & & $X$ & & & & $\mathrm{X}$ & & & $X$ \\
\hline $\begin{array}{l}\text { Lozano- } \\
\text { Fuentes, et } \\
\text { al. }^{[37]}\end{array}$ & DDSS (Mexico) & 2008 & $X$ & & & & & & & & $\mathrm{X}$ & & & $X$ \\
\hline $\begin{array}{l}\text { Senior and } \\
\text { Copley }{ }^{[40]}\end{array}$ & EPIMS (UK) & 2008 & $X$ & $\mathrm{X}$ & $\mathrm{X}$ & & $\mathrm{X}$ & $\mathrm{X}$ & $\mathrm{X}$ & & $\mathrm{X}$ & $\mathrm{X}$ & $X$ & $X$ \\
\hline $\begin{array}{l}\text { Marti, et } \\
\text { al. }^{[36]}\end{array}$ & $\begin{array}{l}\text { EXPLORIS } \\
\text { (Spain) }\end{array}$ & 2008 & $X$ & & & & $\mathrm{X}$ & & & & & & & $\mathrm{X}$ \\
\hline $\begin{array}{l}\text { Ding, et } \\
\text { al. }^{[39]}\end{array}$ & $\begin{array}{l}\text { HAZUS-MH } \\
\text { (US) }\end{array}$ & 2008 & $X$ & & & & $X$ & & & & $\mathrm{X}$ & & & $X$ \\
\hline $\begin{array}{l}\text { Gotham, et } \\
\text { al. }^{[34]}\end{array}$ & HERDS (US) & 2007 & $X$ & $X$ & $\mathrm{X}$ & $\mathrm{X}$ & $\mathrm{X}$ & $\mathrm{X}$ & $\mathrm{X}$ & $\mathrm{X}$ & $X$ & $X$ & & $X$ \\
\hline $\begin{array}{l}\text { Chronaki, et } \\
\text { al. }^{[35]}\end{array}$ & SAFE (Greece) & 2007 & $X$ & $X$ & $\mathrm{X}$ & & $\mathrm{X}$ & $\mathrm{X}$ & $\mathrm{X}$ & & & $\mathrm{X}$ & $\mathrm{X}$ & $\mathrm{X}$ \\
\hline $\begin{array}{l}\text { Kimball, et } \\
\text { al. }\end{array}$ & $\begin{array}{l}\text { ACCESS Grid } \\
\text { (US) }\end{array}$ & 2006 & & & $\mathrm{X}$ & & $\mathrm{X}$ & $\mathrm{X}$ & & & & $\mathrm{X}$ & $\mathrm{X}$ & \\
\hline $\begin{array}{l}\text { Hauenstein, } \\
\text { et al. }{ }^{[33]}\end{array}$ & AID-N (US) & 2006 & $X$ & $\mathrm{X}$ & $\mathrm{X}$ & & & X & & & $\mathrm{X}$ & $\mathrm{X}$ & $\mathrm{X}$ & \\
\hline Lee, et al. ${ }^{[31]}$ & RealOpt (US) & 2006 & $X$ & & & $X$ & $X$ & & & & $X$ & & & \\
\hline $\begin{array}{l}\text { Hochstein, } \\
\text { et al. }{ }^{[32]}\end{array}$ & ToxMap (US) & 2006 & $X$ & & & & & & & & & & & $\mathrm{X}$ \\
\hline $\begin{array}{l}\text { Waring, et } \\
\text { al. }{ }^{[28]}\end{array}$ & $\begin{array}{l}\text { Arcview } 3.2 \text { with } \\
2000 \text { Census data } \\
\text { (US) }\end{array}$ & 2005 & $X$ & & & & $X$ & & & X & X & & & $X$ \\
\hline $\begin{array}{l}\text { Aleskerov, } \\
\text { et al. }{ }^{[30]}\end{array}$ & $\begin{array}{l}\text { DSS-DM } \\
\text { (Turkey) }\end{array}$ & 2005 & $X$ & & & $\mathrm{X}$ & $X$ & & & & & & & \\
\hline $\begin{array}{l}\text { Washington, } \\
\text { et al. }{ }^{[27]}\end{array}$ & Maxi-Vac (US) & 2005 & $X$ & & & & $X$ & & & & X & & & \\
\hline
\end{tabular}




\begin{tabular}{|c|c|c|c|c|c|c|c|c|c|c|c|}
\hline $\begin{array}{l}\text { Horney, et } \\
\text { al. }^{[29]}\end{array}$ & $\begin{array}{l}\text { NCCPHP } \\
\text { Training Web } \\
\text { Site (US) } \\
\end{array}$ & 2005 & $X$ & & $X$ & & & & & $\mathrm{X}$ & \\
\hline $\begin{array}{l}\text { Ptochos, et } \\
\text { al. }{ }^{[26]}\end{array}$ & GEPIMI (Greece) & 2004 & $X$ & $X$ & & $\mathrm{X}$ & $X$ & $\mathrm{X}$ & $X$ & & $\mathrm{X}$ \\
\hline $\begin{array}{l}\text { Bedard, et } \\
\text { al. }{ }^{[25]}\end{array}$ & $\begin{array}{l}\text { ICEM-SE Project } \\
\text { (Canada) }\end{array}$ & 2003 & $X$ & $X$ & & $\mathrm{X}$ & & $X$ & & & $\mathrm{X}$ \\
\hline $\begin{array}{l}\text { Eguchi, et } \\
\text { al. }{ }^{[24]}\end{array}$ & EPEDAT (US) & 1997 & $X$ & & & & & & & & $\mathrm{X}$ \\
\hline $\begin{array}{l}\text { O'Carroll, et } \\
\text { al. }{ }^{[23]}\end{array}$ & $\begin{array}{l}\text { Emergency } \\
\text { Computer } \\
\text { Communications } \\
\text { Network (US) } \\
\end{array}$ & 1995 & $X$ & $X$ & & $\mathrm{X}$ & $X$ & & $\mathrm{X}$ & $\mathrm{X}$ & \\
\hline
\end{tabular}

While all the public health continuity of operations information needs described in our list of categories were identified in the entire set of published studies, only two of the twenty included studies (HERDS ${ }^{[34]}$ and SAFE ${ }^{[35]}$ ) supported all of the needs included in our list. Of these, SAFE is a proposed framework and HERDS a fully operational system. As such, HERDS represents an archetypal working system that other public health continuity of operations planning informatics projects should look to for successful system design. That we start to see information about systems like HERDS and SAFE available in the published literature only as recently as 2007 may indicate a growing level of maturity in public health emergency management development efforts and knowledge. Interestingly, the Emergency Computer Communications Network ${ }^{[23]}$, successfully deployed in 1993 and published about in 1995, satisfied many of the information needs that both HERDS and SAFE support using pre-Web technology. As such, it can be judged as an innovative and sophisticated front-runner of current, more "modern" systems.

Systems and technology projects span the range of implementation levels from proposed frameworks based on existing technology to fully operational systems in current use. All are described at some measure of detail that can help to inform the future design of information systems to support public health continuity of operations. Sixteen of twenty projects explicitly note sponsoring agencies or resource contributors. Where not noted, university affiliation has been listed as the sponsoring agency in Table I. In all cases where noted, project sponsoring agencies are government agencies or a combination of government agencies.

\section{Categories of systems}

Based on their descriptions and common patterns of supported information needs, we were able to group the information systems and technology projects from the included studies into four categories of our own creation. These categories are:

- Risk assessment decision support tools

- Networked and communications-enabled decision support tools

- Dedicated training tools 
Use of Technology to Support Information Needs for Continuity of Operations Planning in Public Health: A Systematic Review

- Dedicated video-conferencing tools

Information systems and technology projects are shown grouped by category and the information needs each category supports in Table III.

Table 3. Categories of systems and projects grouped by information needs they support

\begin{tabular}{|c|c|c|}
\hline Category & Supported Information Needs & System Name \\
\hline $\begin{array}{l}\text { Risk assessment } \\
\text { decision support } \\
\text { tools }(\mathrm{n}=12)\end{array}$ & $\begin{array}{l}\text { Risk Assessment decision support tools typically } \\
\text { support the following set of information needs: } \\
\text { synthesized information, planning/plan } \\
\text { testing/exercise support, resource } \\
\text { tracking/capacity management and geographic } \\
\text { data }\end{array}$ & $\begin{array}{l}\text { EPEDAT }^{[24]} \\
\text { ICEM-SE Project }^{[25} \\
\text { Maxi-Vac }^{[27]} \\
\text { Arcview } 3.2 \text { with } \\
\text { 2000 Census data } \\
\text { DSS-DM[28] } \\
\text { RealOpt }^{[31]} \\
\text { ToxMap }^{[32]} \\
\text { EXPLORIS }^{[36]} \\
\text { DDSS }^{[37]} \\
\text { BRFSS + GIS }_{\text {Technology }}^{[37]} \\
\text { HAZUS-MH }^{[39]} \\
\text { BCMS }^{[41]}\end{array}$ \\
\hline $\begin{array}{l}\text { Networked and } \\
\text { communications- } \\
\text { enabled decision } \\
\text { support tools }(n=6)\end{array}$ & $\begin{array}{l}\text { Networked and communications-enabled decision } \\
\text { support tools typically support the following set of } \\
\text { information needs: synthesized information, } \\
\text { centralized data access, coordination/incident } \\
\text { command support, planning/plan testing/exercise } \\
\text { support, interoperable external } \\
\text { communication/alerting, resource } \\
\text { tracking/capacity management, collaboration, } \\
\text { remote work/portable data }\end{array}$ & $\begin{array}{l}\text { Emergency } \\
\text { Computer } \\
\text { Communications } \\
\text { Network }(\mathrm{CDC} \\
\text { Wonder/PC })^{[23]} \\
\text { GEPIMI }^{[26]} \\
\text { AID-N }^{[33]} \\
\text { HERDS }^{[34]} \\
\text { SAFE }^{[35]} \\
\text { EPIMS }^{[40]}\end{array}$ \\
\hline $\begin{array}{l}\text { Dedicated training } \\
\text { tools }(\mathrm{n}=1)\end{array}$ & These tools are dedicated specifically to training & $\begin{array}{l}\text { NCCPHP Training } \\
\text { Web Site }^{[29]}\end{array}$ \\
\hline $\begin{array}{l}\text { Dedicated video- } \\
\text { conferencing tools } \\
(\mathrm{n}=1)\end{array}$ & $\begin{array}{l}\text { These tools are dedicated specifically to } \\
\text { maintaining a video-conference platform }\end{array}$ & Access Grid $^{[22]}$ \\
\hline
\end{tabular}




\section{Follow-up to initial search}

We repeated our search using the same methodology and terms in February of 2010 for the period between January 2009 to February 2010 in order to provide coverage for time that passed during data analysis and the writing of this manuscript. Thirty-nine publications were returned in the results set for this search. Of these, one article ${ }^{[42]}$ was a follow-up to an included business continuity videoconferencing article already included in the review ${ }^{[22]}$. In addition, three studies $^{[43-45]}$ demonstrated methods that combined GIS technology and public health data but did not meet our inclusion/exclusion criteria. All three studies were published in the International Journal of Health Geographics, a newer journal dedicated to the application of health-related GIS. This journal and these articles may indicate a trend that GIS technology has an expanded role to play in the design of future public health COOP information systems. This finding suggests a full review of current use of GIS technologies in public health may be appropriate. However, such an undertaking is beyond the scope of this project. We include references to the following articles ${ }^{[46-51]}$ and books ${ }^{[52-54]}$ as sources for those interested in the application of GIS to public health. For those interested in the application of GIS to disaster management we offer references to the following books. ${ }^{[55-57]}$

\section{Limitations}

One limitation of this systematic review is that it relies on a list of information needs based on author familiarity with a corpus of literature gained through searches over time coupled with author experience gained through information design research with public health continuity of operations planners rather than an exhaustive list of information needs compiled through a formal literature review. In addition, this review does not undertake a formal analysis of the differential use of the same or similar terms across disciplines although we believe that this limitation is addressed by the divergent approach in the search term selection and broad inclusion criteria applied to the set of documents returned as a result of the search process.

\section{Implications for future research}

This review will raise awareness of the importance of continuity of operations planning in public health as well as the attendant need for integrated systems to support public health COOP. In addition, these findings can help public health informaticians in defining the types of systems that need to be designed and developed to support public health operational continuity.

\section{Conclusion}

While there have been a number of recent efforts to develop information systems that support public health emergency management, overall, we lack documented efforts of information systems that support public health continuity of operations planning. Consequently, we lack much evidence of what does and does not work in this area of research. One quick and simple way to raise awareness of the COOP topic for researchers might be to index emergency 
management technology studies with COOP and BC key words. However, while public health practitioners involved in continuity of operations planning and emergency management activities may have similar information needs before, during and after a crisis, the goals of these activities are different and, indeed, they compete with each other for organizational resources. As such, determining design specifications for information technology-based COOP systems should involve public health practitioners who engage in COOP activities as part of their work flow to ensure support of their information needs. Though there are common themes (as highlighted in this paper) between public health COOP and emergency response, some COOP activities in the public health domain are unique and technology designed to support these activities must be context-specific to address the uniqueness of the environment in which they are introduced.

\section{Conflicts of interest}

The authors have no conflicts of interest to report at this time.

\section{Acknowledgments}

This study was supported in part by the National Library of Medicine Medical Informatics Training Grant T15 LM007442-07.

\section{Correspondence}

Blaine Reeder: breeder@u.washington.edu

\section{References}

[1]. Rozek P, Groth D. Business continuity planning. It's a critical element of disaster preparedness. Can you afford to keep it off your radar? Health Manag Technol 2008 Mar;29(3):10-2.

[2]. Dawes SS, Cresswell AM, Cahan BB. Learning From Crisis: Lessons in Human and Information Infrastructure From the World Trade Center Response. Social Science Computer Review 2004;22(1):52-66.

[3]. Somers S. Survey and Assessment of Planning for Operational Continuity in Public Works. PUBLIC WORKS MANAGEMENT AND POLICY 2007;12(2):451-64.

[4]. Buehler JW, Whitney EA, Berkelman RL. Business and public health collaboration for emergency preparedness in Georgia: a case study. BMC Public Health 2006;6:285.

[5]. Reeder B, Demiris G. Building the PHARAOH Framework Using Scenario-Based Design: A Set of Pandemic Decision-Making Scenarios for Continuity of Operations in a Large Municipal Public Health Agency. Journal of Medical Systems.

[6]. Fraser MR. After 5 Years of Public Health Preparedness, Are We Ready Yet? Journal of public health management and practice : JPHMP 2007;13(1):3-6.

[7]. Reeder B, Demiris G, editors. Exploring the role of Information Technology in DecisionMaking of Business Continuity Planners in Public Health (Poster Presentation). Robert Wood 
Johnson Foundation Public Health Informatics Fellows Workshop; 2008 July 7, 2008; Hilton Washington DC/Rockville Executive Meeting Center.

[8]. Revere D, Turner AM, Madhavan A, Rambo N, Bugni PF, Kimball A, Fuller SS.

Understanding the information needs of public health practitioners: a literature review to inform design of an interactive digital knowledge management system. J Biomed Inform 2007 Aug;40(4):410-21.

[9]. Cerullo V, Cerullo MJ. Business Continuity Planning: A Comprehensive Approach. INFORMATION SYSTEMS MANAGEMENT 2004;21(3):70-8.

[10]. Grimaldi RJ. Why Do Business Continuity Plans Fail? Risk management 2002;49:34-9. [11]. Whitworth PM. Continuity of Operations Plans: Maintaining Essential Agency Functions When Disaster Strikes. Journal of Park \& Recreation Administration: Sagamore Publishing, LLC; 2006. p. 40-63.

[12]. Rebmann T, Carrico R, English JF. Lessons public health professionals learned from past disasters. Public health nursing (Boston, Mass) 2008;25(4).

[13]. Rogers B, Lawhorn E. Disaster Preparedness: Occupational and Environmental Health Professionals' Response to Hurricanes Katrina and Rita. AAOHN JOURNAL 2007;55(5):197210.

[14]. Gallo RJ, Campbell D. Bioterrorism: challenges and opportunities for local health departments. Journal of public health management and practice : JPHMP 2000;6(4):57-62. [15]. Kuntz S, Smilie J, Wang J. How Ready Are We? Measuring Montana's Statewide Public Health Preparedness. Northwest Public Health. Northwest Public Health 2005;22(2):2. [16]. Mann NC, MacKenzie E, Anderson C. Public Health Preparedness for Mass-Casualty Events: A 2002 State-by-State Assessment. PREHOSPITAL AND DISASTER MEDICINE 2004;19(3):245-55.

[17]. Morse A. Bioterrorism preparedness for local health departments. Journal of community health nursing 2002;19(4):203-11.

[18]. Rodriguez H, Aguirre BE. Hurricane Katrina and the Healthcare Infrastructure: A Focus on Disaster Preparedness, Response, and Resiliency. Frontiers of health services management 2006;23(1):13-24.

[19]. Briggs SM. Disaster management teams. CURRENT OPINION IN CRITICAL CARE 2005;11(6):585-9.

[20]. Perry RW. Disaster Exercise Outcomes for Professional Emergency Personnel and Citizen Volunteers. Journal of Contingencies and Crisis Management 2004;12(2):64-75.

[21]. Shover $\mathrm{H}$. Understanding the chain of communication during a disaster. Perspectives in psychiatric care 2007;43(1):4-14.

[22]. Kimball AM, Arima Y, Osaki C, Werle J, Kobayashi J, Brown J, Fox L. Real-time videoconferencing: promise for pandemic influenza preparedness. Journal of Telemedicine \& Telecare. [Article]. 2006;12:53-5.

[23]. O'Carroll PW, Friede A, Noji EK, Lillibridge SR, Fries DJ, Atchison CG. The Rapid Implementation of a Statewide Emergency Health Information System During the 1993 Iowa Flood. American Journal of Public Health. [Article]. 1995;85:564-7.

[24]. Eguchi RT, Goltz JD, Seligson HA, Flores PJ, Blais NC, Heaton TH, Bortugno E. RealTime Loss Estimation as an Emergency Response Decision Support System: The Early PostEarthquake Damage Assessment Tool (EPEDAT). Earthquake Spectra 1997;13(4):815-32. 
[25]. Bedard Y, Gosselin P, Rivest S, Proulx MJ, Nadeau M, Lebel G, Gagnon MF. Integrating GIS components with knowledge discovery technology for environmental health decision support. Int J Med Inform 2003 Apr;70(1):79-94.

[26]. Ptochos D, Panopoulos D, Metaxiotis K, Askounis D. Using internet GIS technology for early warning, response and controlling the quality of the public health sector. Int J Electron Healthc 2004;1(1):78-102.

[27]. Washington ML, Mason J, Meltzer MI. Maxi-Vac: planning mass smallpox vaccination clinics. Journal of Public Health Management \& Practice 2005;11(6):542-9.

[28]. Waring S, Zakos-Feliberti A, Wood R, Stone M, Padgett P, Arafat R. The utility of geographic information systems (GIS) in rapid epidemiological assessments following weatherrelated disasters: Methodological issues based on the Tropical Storm Allison experience. International Journal of Hygiene \& Environmental Health. [Article]. 2005;208:109-16. [29]. Horney JA, MacDonald P, Rothney EE, Alexander LK. User patterns and satisfaction with on-line trainings completed on the North Carolina Center for Public Health Preparedness Training Web Site. J Public Health Manag Pract 2005 Nov;Suppl:S90-4.

[30]. Aleskerov F, Say AI, Toker Al, Akin HL, Altay Gl. A cluster-based decision support system for estimating earthquake damage and casualties. Disasters. [Article]. 2005;29:255-76. [31]. Lee EK, Maheshwary S, Mason J, Glisson W. Decision support system for mass dispensing of medications for infectious disease outbreaks and bioterrorist attacks. Annals of Operations Research. [Article]. 2006;148:25-53.

[32]. Hochstein C, Szczurm M. TOXMAP: A GIS-Based Gateway to Environmental Health Resources. Medical Reference Services Quarterly. [Article]. 2006 Fall2006;25:13-31.

[33]. Hauenstein L, Gao T, Sze TW, Crawford D, Alm A, White D. A cross-functional serviceoriented architecture to support real-time information exchange in emergency medical response. Conf Proc IEEE Eng Med Biol Soc 2006;Suppl:6478-81.

[34]. Gotham IJ, Sottolano DL, Hennessy ME, Napoli JP, Dobkins G, Le LH, Burhans RL, Fage BI. An integrated information system for all-hazards health preparedness and response: New York State Health Emergency Response Data System. J Public Health Manag Pract 2007 SepOct;13(5):486-96.

[35]. Chronaki CE, Berthier A, Lleo MM, Esterle L, Lenglet A, Simon F, Josseran L, Lafaye M, Matsakis Y, Tabasco A, Braak L. A satellite infrastructure for health early warning in postdisaster health management. Stud Health Technol Inform 2007;129(Pt 1):87-91.

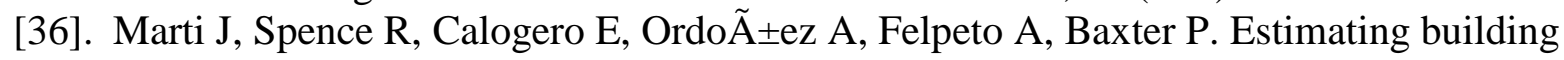
exposure and impact to volcanic hazards in Icod de los Vinos, Tenerife (Canary Islands). Journal of Volcanology \& Geothermal Research. [Article]. 2008;178:553-61.

[37]. Lozano-Fuentes S, Elizondo-Quiroga D, Farfan-Ale JA, Lorono-Pino MA, Garcia-Rejon J, Gomez-Carro S, Lira-Zumbardo V, Najera-Vazquez R, Fernandez-Salas I, Calderon-Martinez J, Dominguez-Galera M, Mis-Avila P, Morris N, Coleman M, Moore CG, Beaty BJ, Eisen L. Use of Google Earth to strengthen public health capacity and facilitate management of vector-borne diseases in resource-poor environments. Bull World Health Organ 2008 Sep;86(9):718-25. [38]. Holt JB, Mokdad AH, Ford ES, Simoes EJ, Mensah GA, Bartoli WP. Use of BRFSS data and GIS technology for rapid public health response during natural disasters. Prev Chronic Dis $2008 \mathrm{Jul} ; 5(3)$ :A97.

[39]. Ding A, White JF, Ullman PW, Fashokun AO. Evaluation of HAZUS-MH Flood Model with Local Data and Other Program. Natural Hazards Review 2008;9(1):20-8. 
[40]. Senior A, Copley R. Deploying a new system for recording and managing information during an emergency to aid decision making. JOURNAL OF BUSINESS CONTINUITY AND EMERGENCY PLANNING 2008;2(3):267-80.

[41]. Sheth S, McHugh J, Jones F. A dashboard for measuring capability when designing, implementing and validating business continuity and disaster recovery projects. JOURNAL OF BUSINESS CONTINUITY AND EMERGENCY PLANNING 2008;2(3):221-39. [42]. Kimball AM, Arima Y, French HM, Osaki CS, Hoff R, Lee SS, Schafer L, Nabae K, Hsun C, Hishamuddin P, Nelson R, Woody K, Brown J, Fox L. Pandemic influenza planning by videoconference. J Telemed Telecare 2009;15(7):368-72. [43]. Bernier E, Gosselin P, Badard T, Bedard Y. Easier surveillance of climate-related health vulnerabilities through a Web-based spatial OLAP application. Int J Health Geogr 2009;8(18):18.

[44]. Kamadjeu R. Tracking the polio virus down the Congo River: a case study on the use of Google Earth in public health planning and mapping. Int J Health Geogr 2009;8(4):4.

[45]. Noor AM, Alegana VA, Gething PW, Snow RW. A spatial national health facility database for public health sector planning in Kenya in 2008. Int J Health Geogr 2009;8(13):13.

[46]. Cromley EK. GIS AND DISEASE. Annual Review of Public Health: Annual Reviews Inc.; 2003. p. 7.

[47]. Croner CM. PUBLIC HEALTH, GIS, AND THE INTERNET. Annual Review of Public Health: Annual Reviews Inc.; 2003. p. 57.

[48]. Khan OA, NetLibrary I. Geographic information systems and health applications. Hershey, PA: Idea Group Pub.; 2003. [49]. McLafferty SL. GIS AND HEALTH CARE. Annual Review of Public Health: Annual Reviews Inc.; 2003. p. 25. [50]. Ricketts TC. GEOGRAPHIC INFORMATION SYSTEMS AND PUBLIC HEALTH. Annual Review of Public Health: Annual Reviews Inc.; 2003. p. 1. [51]. Rushton G. PUBLIC HEALTH, GIS, AND SPATIAL ANALYTIC TOOLS. Annual Review of Public Health: Annual Reviews Inc.; 2003. p. 43.

[52]. Cromley EK, McLafferty S. GIS and public health. New York: Guilford Press; 2002.

[53]. Melnick AL. Introduction to geographic information systems in public health.

Gaithersburg, Md.: Aspen Publishers; 2002.

[54]. Maheswaran R, Craglia M. GIS in public health practice. Boca Raton: CRC Press; 2004.

[55]. Amdahl G. Disaster response : GIS for public safety. Redlands, Calif.: ESRI Press; 2001.

[56]. Greene RW. Confronting catastrophe : a GIS handbook. Redlands, Calif.: ESRI Press; 2002.

[57]. Kataoka M. GIS for homeland security. Redlands, Calif.: ESRI Press; 2007. 